\title{
BMJ Open Characteristics of Chinese patients with genetic CJD who have E196A or E196K mutation in PRNP: comparative analysis of patients identified in the Chinese National CJD Surveillance System
}

Qi Shi (1) , ${ }^{1,2}$ Kang Xiao, ${ }^{1}$ Cao Chen, ${ }^{1,3}$ Wei Zhou, ${ }^{1}$ Li-Ping Gao, ${ }^{1}$ Yue-Zhang Wu, ${ }^{1}$ Yuan Wang, ${ }^{1}$ Chao Hu, ${ }^{1}$ Chen Gao, ${ }^{1}$ Xiao-Ping Dong (i) ${ }^{1,3,4}$

To cite: Shi Q, Xiao K, Chen C, et al. Characteristics of Chinese patients with genetic CJD who have E196A or E196K mutation in PRNP: comparative analysis of patients identified in the Chinese National CJD Surveillance System. BMJ Open 2021;11:e054551. doi:10.1136/ bmjopen-2021-054551

- Prepublication history for this paper is available online. To view these files, please visit the journal online (http://dx.doi. org/10.1136/bmjopen-2021054551).

Received 23 June 2021 Accepted 09 August 2021
Check for updates

(C) Author(s) (or their employer(s)) 2021. Re-use permitted under CC BY-NC. No commercial re-use. See rights and permissions. Published by BMJ.

For numbered affiliations see end of article.

Correspondence to Dr Xiao-Ping Dong; dongxp238@sina.com

\section{ABSTRACT}

Objective Two different mutations at codon 196, namely E196A and E196K, have been reported to be related to genetic Creutzfeldt-Jakob disease (CJD). We aimed to comparatively analyse the features of Chinese patients with these two mutations from the CJD surveillance system in China.

Design and setting Comparative analysis of patients identified via the Chinese National CJD Surveillance System during the period 2006-2020.

Participants 16 Chinese patients with genetic CJD with E196A mutation and 5 with E196K mutation.

Methods Neurological examination, EEG and MRI, western blot, gene sequence, and RT-QulC.

Results The age of onset of E196K genetic CJD cases (median of 61 years) was older than the E196A cases (median of 67 years). Generally, these two subtypes of genetic CJD were more like sporadic Creutzfeldt-Jakob disease (sCJD) clinically. The E196A cases showed more major symptoms, while those of E196K cases were restricted to dementia and mental problems. During progression, more SCJD-associated symptoms and signs gradually appeared, but none of the E196K cases showed cerebellum and visual disturbances. Typical periodic sharp wave complexes on MRI were recorded in $25 \%$ of E196A cases but not in E196K cases. SCJD-associated abnormalities on MRI, positive cerebrospinal fluid (CSF) 14-3-3 and increased CSF total tau were observed frequently, ranging from two out of three cases to four out of five cases, without a difference. Positive CSF RT-QulC was detected in $37.5 \%$ (6 of 16) of E196A cases and $60 \%$ (3 of 5) of E196K cases. The duration of survival of E196K cases (median of 4.5 months) was shorter than the E196A cases (median of 6.5 months). Moreover, female cases and cases with young age of onset (<60 years) in E196A displayed longer survival time than male patients and cases with older age of onset ( $\geq 60$ years).

Conclusions This is the largest comprehensive report of genetic CJD with mutations at codon 196 to date, describing the similarity and diversity in clinical and laboratory tests between patients with E196A and with E196K mutations.
Strengths and limitations of this study

- This is the largest comprehensive report of genetic Creutzfeldt-Jakob disease (gCJD) with mutations at codon 196 to date.

- The study provides clinical, genetic and laboratory data on E196A and E196K gCJD, which is a rare disease.

- Mutations in E196K have been described in Caucasians and Asians, while mutations in E196A seem to be reported only in Chinese.

- There was a limited number of enrolled patients $(\mathrm{N}=21)$ in the data set, especially of cases with E196K mutation, which may have resulted in observational bias.

- The pathogenic prion protein $\left(\mathrm{PrP}^{\mathrm{Sc}}\right)$ and neuropathological features of Chinese patients with E196A and E196K gCJD remain unclear due to lack of brain specimens from patients.

\section{INTRODUCTION}

Genetic human prion diseases, caused by different mutations in the prion protein $(\operatorname{PrP})$ encoding gene PRNP, account for approximately $10 \%-15 \%$ of human prion diseases. Genetic human prion diseases have various medical terms based on their clinical and neuropathological phenotypes, that is, genetic Creutzfeldt-Jakob disease (gCJD), GerstmannStraussler-Scheinker syndrome and fatal familial insomnia, which are closely related to different mutations within $P R N P{ }^{12}$ In the context of one same disease term, such as gCJD, the clinical, neuropathological and laboratory features may vary according to mutations at different positions. ${ }^{34}$ Different mutations at the same position lead to substitution of different amino acids, subsequently displaying different phenotypical features, for example, mutations at codons 105, 188 and $196 .^{4}$ 
Two different mutations at codon 196 have been reported to be related to gCJD: E196A and E196K. ${ }^{4-8}$ In a surveillance activity conducted by the Chinese National Surveillance for CJD (CNS-CJD) in Chinese mainland, 16 Chinese gCJD cases with E196A mutation and 5 cases with E196K mutation have been identified in the past 10 years. ${ }^{9}{ }^{10}$ Among them, E196A gCJD was the fourth most frequently observed genetic prion disease in China (Shi et al, unpublished data, 2020). In this study we comparatively analysed the clinical and laboratory characteristics of Chinese gCJD cases with E196A or E196K mutation. Both types of E196 gCJD revealed sporadic CreutzfeldtJakob disease (sCJD) like phenotype in general, while showing differences in some clinical and laboratory features.

\section{MATERIALS AND METHODS \\ Data collection}

As described previously, ${ }^{10}{ }^{11}$ clinical data of patients suspected of CJD were collected by neurologists at the local hospitals while epidemiological data were collected by staff from the local provincial Chinese Centers for Disease Control and Prevention (CDCs) from 2006 to 2020. The final diagnosis was given by an expert team consisting of neurologists, epidemiologists and laboratory staff based on the diagnostic criteria for CJD issued by the Chinese National Health Commission in 2017. Follow-up surveys of patients were conducted by the staff at the centre of CNS-CJD via telephone and/or WeChat.

\section{Western blot for 14-3-3 protein in CSF}

Cerebrospinal fluid (CSF) samples were mixed with $5 \times$ loading buffer and boiled for $8 \mathrm{~min}$. Proteins were separated in $15 \%$ sodium dodecyl sulfonate (SDS)polyacrylamide gel electrophoresis (PAGE) and electronically transferred onto nitrocellulose membranes (Whatman, Pittsburgh, Pennsylvania, USA) by semidry method in a transfer buffer and immunoblotted with anti-14-3-3 polyclonal antibody (1:1000 dilution; Santa Cruz Biological). Reactive signals were visualised using an enhanced chemiluminescence kit (Amersham Pharmacia Biotech, Piscataway, New Jersey, USA).

\section{PCR and sequencing assays for the PRNP gene}

Genomic DNA was extracted from peripheral blood leucocytes using a commercial kit (QIAGEN, Germany). One hundred nanograms of the extracted DNA were amplified by PCR using specific PRNP primers (forward primer: 5'-GGC AAA CCT TGG ATG CTG G-3' and reverse primer: 5 '-CCC ACT ATC AGG AAG ATG AGG3'). ${ }^{10}$ Sequencing analysis of the PRNP gene was conducted according to standard operating procedure. ${ }^{10}$

\section{ELISA for total tau in CSF}

The amount of protein tau in the CSF samples was quantitatively measured by a commercial ELISA kit (81572; Innotest hTau-Ag, Belgium). Briefly, ${ }^{12} 25 \mu \mathrm{L}$ of the CSF sample were diluted with the buffer supplied by the manufacturer and added to the wells of the antibody-coated plate in duplicate. The plate was incubated at room temperature (RT) overnight. After washing five times, $100 \mu \mathrm{L}$ of peroxidase, horseradish (HRP)-conjugated detection antibodies were added to each well and incubated at RT for $30 \mathrm{~min}$. Absorbance at $450 \mathrm{~nm}$ in each well was measured by a microplate reader (Perkin Elmer, USA) after developing with $100 \mu \mathrm{L}$ substrate working solution for $30 \mathrm{~min}$ in dark and terminated with $2 \mathrm{M} \mathrm{H}_{2} \mathrm{SO}_{4}$. Tau concentrations in the tested CSF samples were calculated based on a tau standard curve.

\section{RT-QuiC assays}

Real-time quaking induced conversion (RT-QuIC) assay was performed according to the working procedures described previously. ${ }^{13}$ Briefly, each reaction contained $10 \mu \mathrm{g}$ of a recombinant hamster protein ( $\mathrm{rHaPrP90}-231)$, 1X phosphate buffer saline (PBS), $170 \mathrm{mM} \mathrm{NaCl}, 1 \mathrm{mM}$ EDTA, $0.01 \mathrm{mM}$ thioflavin $\mathrm{T}$ (ThT) and $0.001 \%$ SDS, together with $15 \mu \mathrm{L}$ of the CSF sample, in a final volume of $100 \mu \mathrm{L}$. The assay was conducted in a black 96-well, optical-bottomed plate (Nunc, 265301) on a BMG FLUOstar Plate Reader (BMG LABTECH). The following were the working conditions: temperature, $55^{\circ} \mathrm{C}$; vibration speed, 700 revolution per minute $(\mathrm{rpm})$; vibration/incubation time, $60 / 60 \mathrm{~s}$; and total reaction time, 60 hours. The ThT fluorescence value (excitation wavelength, $450 \mathrm{~nm}$; emission wavelength, $480 \mathrm{~nm}$ ) of each reaction was automatically counted every $45 \mathrm{~min}$ and further presented as relative fluorescence units. Each sample was tested in quadruplicate simultaneously. The cut-off value was set as the mean value of the negative controls plus 10 times the SD. A sample was considered to be positive when $\geq 2$ wells revealed positive reaction curves. A $10^{-5}$ diluted brain homogenate of the scrapie agent 263K-infected hamster was used as the positive control, while $10^{-5}$ diluted brain homogenate of the normal hamster was used as the negative control.

\section{Statistical assays}

Statistical analyses were performed using the SPSS V.11.5 statistical software program.

\section{Patient and public involvement}

No patients were involved.

\section{RESULTS}

\section{General information}

Since 2006, more than 200 cases of genetic human prion diseases have been identified and diagnosed via CNSCJD, which consisted of 19 different subtypes of mutations in PRNP. ${ }^{9}{ }^{10}$ Among them, 16 patients with E196A gCJD and 5 patients with E196K gCJD were identified via the Chinese National CJD Surveillance System during the period 2006-2020. The first E196A and E196K gCJD cases were reported in 2011 and 2009, respectively (figure 1A). 

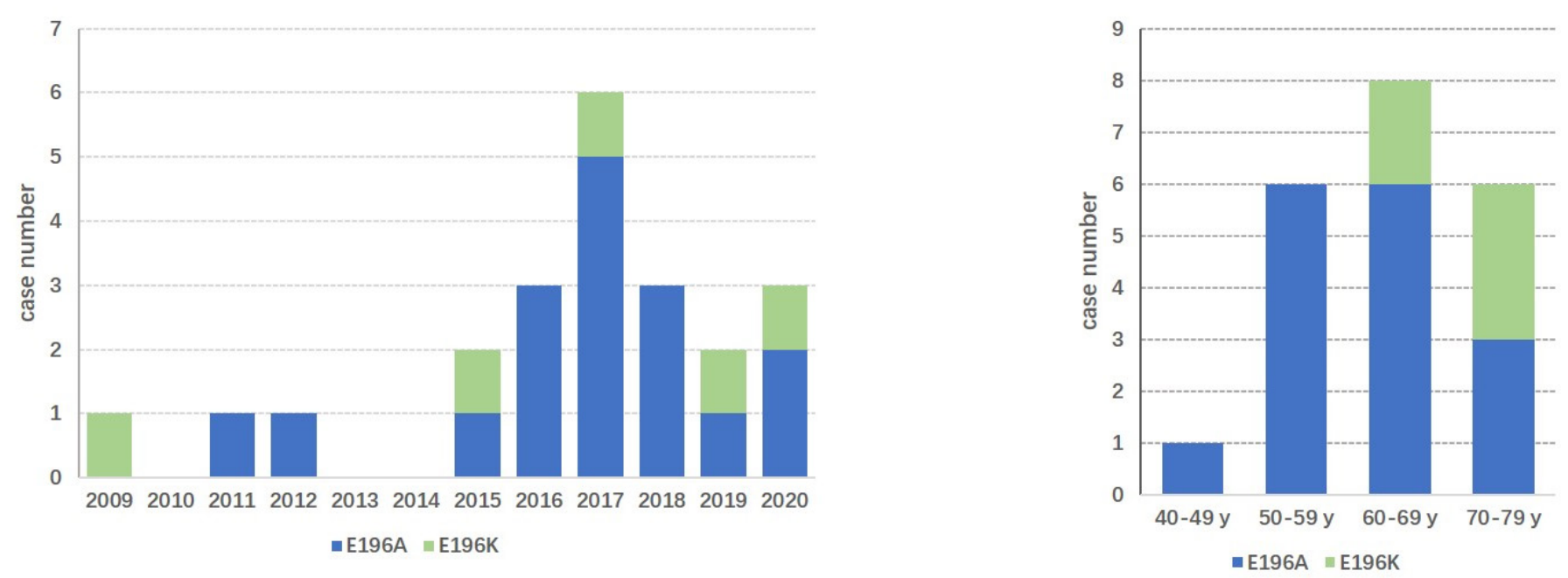

Figure 1 Distribution of Chinese patients with E196A and E196K genetic Creutzfeldt-Jakob disease based on (A) year of diagnosis and $(B)$ age of onset.

Afterwards, more cases of E196A gCJD were diagnosed, particularly since 2015, and peaked in 2017. E196K gCJD cases were markedly less frequent, with one case reported in 2015, 2017, 2019 and 2020, respectively. The gender (male:female) distribution of E196A and E196K cases was 1:0.78 (9:7) and 1:1.5 (2:3). The age of onset of E196A cases varied from 43 to 76 years old, with a median of 61 years, while that of E196K cases varied from 61 to 77 years old, with a median of 67 years. The peak age of onset in E196A patients was 50-59 years and 60-69 years, which looked younger than that of E196K patients (figure 1B). Analysis of age of onset of patients based on gender found that the median age of onset among E196A male patients was older than of female patients (65 years vs 56 years), while the median age of onset among E196K male patients was younger than of female patients (62.5 years vs 73.5 years). No significant geographical-associated and occupational-associated phenomenon was observed.

\section{Clinical features}

The clinical, genetic and laboratory data of 16 cases of E196A gCJD and 5 cases of E196K gCJD are summarised in table 1 . The intervals from onset to diagnosis varied largely, ranging from 1 to 13 months. Majority of the patients (18 of 21) were diagnosed within 6 months of onset, without notable differences between the E196A and E196K groups. Most data on clinical manifestations, examinations and laboratory tests of these patients were obtained during the period of hospitalisation and were referred to CNS-CJD. Some information was collected via follow-up surveys after discharge. Patients with E196A mutation displayed two to four major symptoms (table 2). Dementia (cognitive decline and memory loss) was the complaint in $68.8 \%$ (11 of 16) of cases, followed by mental problems (emotional lability and anxiety) in $62.5 \%$ (10 of 14), extrapyramidal dysfunction (unsteady gait, drooling and shaking of limbs) in $43.8 \%$ (7 of 16) and cerebellum disorder (ataxia, speech dysgraphia and dysmetria) in $43.8 \%$ (7 of 16). Three patients described cortical blindness and one complained of paraesthesia. A slight difference in major symptoms was observed between gender and between young ( $<60$ years) and elderly ( $>60$ years) patients, but without statistical significance. In contrast, five patients with E196K mutation showed fewer initial disorders, limited to dementia (4 of 5) and mental problems (2 of 5 ). Other symptoms were rarely recorded.

Along with progression, rapid progressive dementia was reported in all patients regardless of mutation, E196A or E196K. Other sCJD-associated symptoms and signs were also observed gradually. In the group of patients with E196A mutation, five patients were recorded to have four major sCJD-associated symptoms, seven cases with three symptoms and three cases with two symptoms. Only one case (case 9) did not show the four major symptoms, although the patient had clear mental problems (table 1 ). The rates of detection of myoclonus, cerebellum and visual disorders, pyramidal and extrapyramidal symptoms, and mutism were $68.8 \%$ (11 of 16), 81.3 (13 of $16), 87.5 \%$ (14 of 16 ) and $62.5 \%$ (10 of 16 ), respectively (figure 2A). In the group of patients with E196K mutation, all five patients showed myoclonic movement, while none reported cerebellum and visual disorders. Four cases displayed pyramidal and extrapyramidal symptoms and two had mutism (table 1 and figure 2B).

\section{EEG and MRI features}

All patients received electroencephalogram (EEG) and MRI examinations at least one time. In the group of patients with E196A mutation, four patients recorded typical periodic sharp wave complexes (PSWCs) on EEG, seven showed different abnormalities but without PSWCs, and another five cases had uncertain PSWCs (table 1 and 


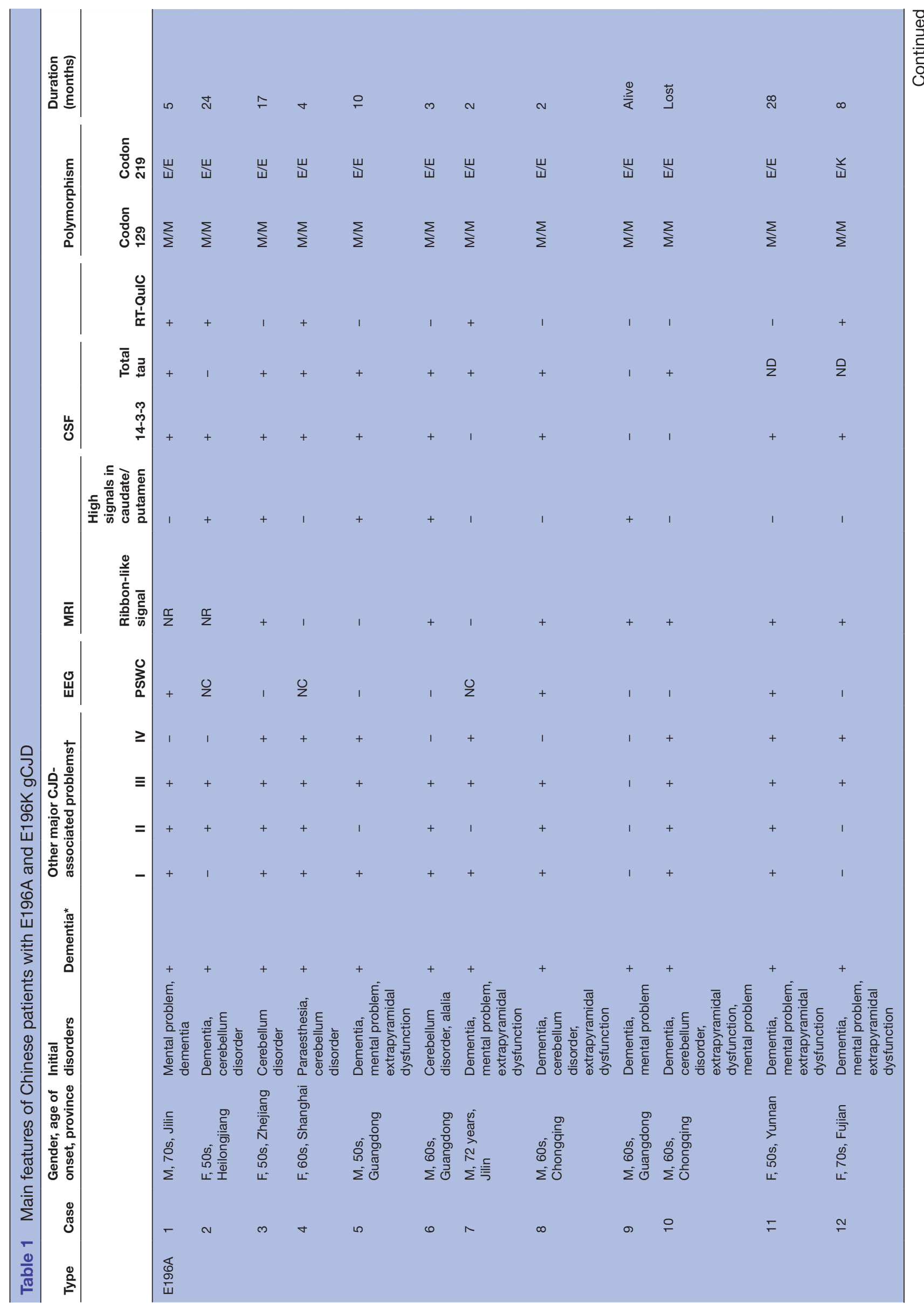




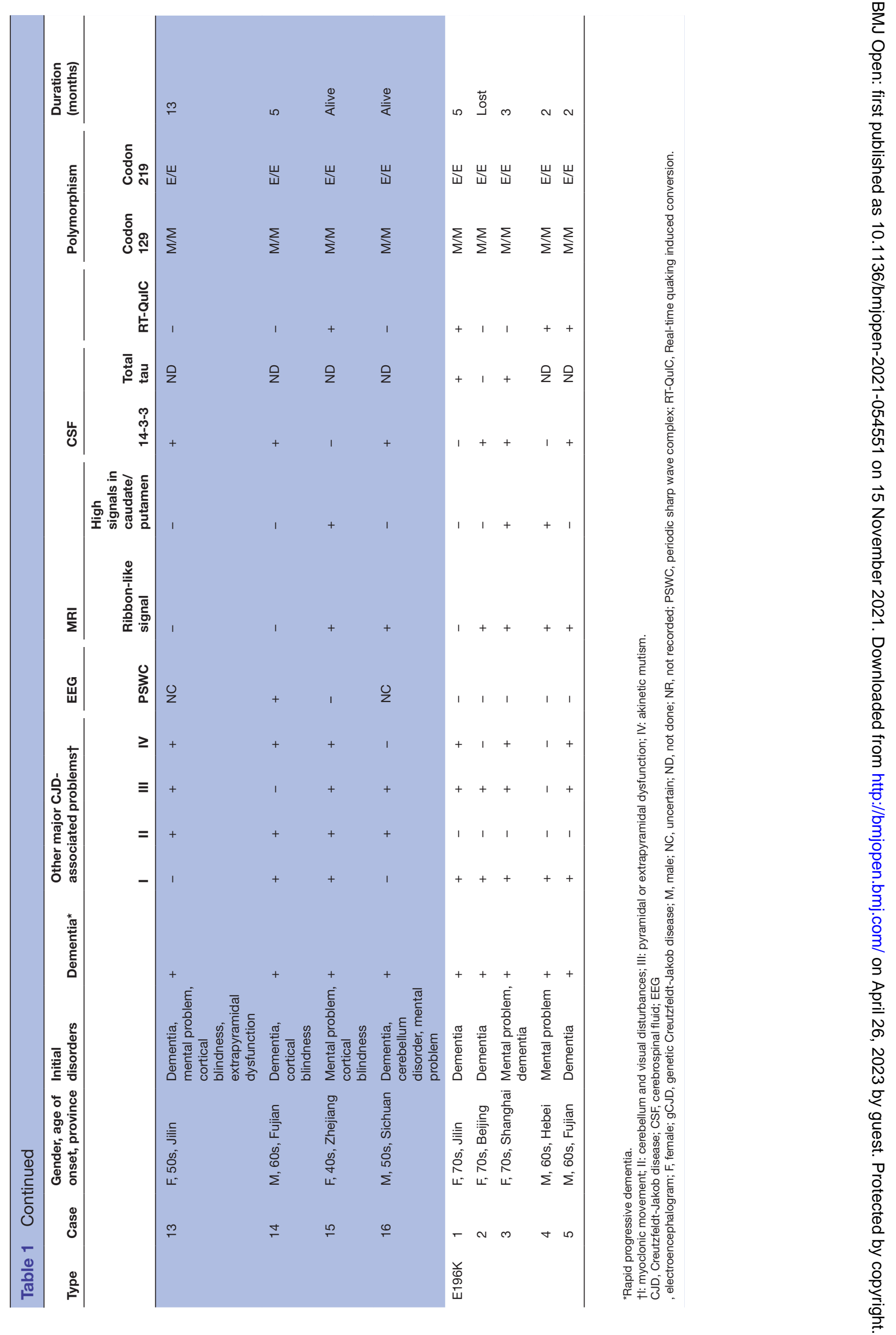


Table 2 Major symptoms of patients with genetic Creutzfeldt-Jakob disease with E196A or E196K mutation

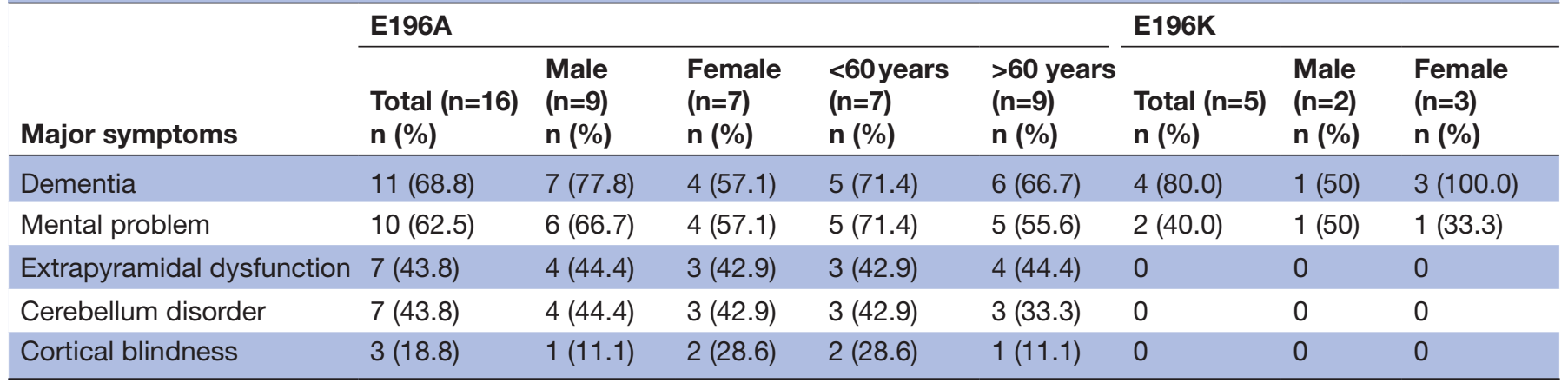

figure $3 \mathrm{~A})$. The positivity rate of definite PSWC on EEG in $\mathrm{E} 196 \mathrm{~A}$ cases was $25 \%$. In the group of patients with E196K, none of the five patients showed typical PSWCs (figure 3A). The sCJD-associated MRI abnormalities (ribbon-like signal on diffusion weighted imaging (DWI) and/or high signals in the caudate/putamen) were observed in $68.8 \%$ (11 of 16 ) of E196A cases and $80 \%$ (4 of 5) of E196K cases (figure 3B). Ribbon-like signals (9 out of 14 cases with E196A and 4 with E196K) were more frequently detected than high signals in the caudate/ putamen (6 cases with E196A and 2 cases with E196K) (table 1).

\section{PRNP gene sequencing}

Mutations at codon 196 in one PRNPallele of the patients were verified by direct sequencing of the PCR products, which was routinely repeated at least two times with newly extracted DNAs. Sixteen suspected CJD cases contained a missense mutation at codon 196 of the PRNP gene, leading to a substitution of glutamic acid by alanine, while five cases had a mutation causing substitution of glutamic acid by lysine. No additional nucleotide exchanges were found in other regions of the PRNP sequences of those cases. All patients had methionine homozygosity at codon 129 (M129M). Out of 16 E196A cases, 15 had glutamic acid homozygosity at codon 219 (E219E) and 1 case (case
12) had glutamic acid/lysine heterozygosity (E219K). All five E196K cases had the sequencing data of codon 219, revealing E219E.

\section{CSF protein 14-3-3 and tau}

Lumbar puncture was conducted in all patients. Routine items (cells, proteins, glucose, electrolytes, etc) on CSF biochemistry were all in the normal ranges. Western blots for CSF 14-3-3 were positive in 75\% (12 of 16) of E196A cases and 60\% (3 of 5 ) of E196K cases (figure 3C). The total tau level in the CSF samples from 10 cases with E196A and 3 cases with E196K (table 1) was measured with a commercial ELISA kit and a tau level higher than $1400 \mathrm{pg} / \mathrm{mL}$ was considered positive based on previous studies. ${ }^{1214}$ Eight (out of ten) cases with E196A mutation and two (out of three) cases with E196K mutation were positive (figure 3D).

\section{RT-QulC features}

All cases with E196A and E196K mutations in this study were subjected to RT-QuIC tests with $15 \mu \mathrm{L}$ CSF sample each, using a recombinant truncated hamster PrP protein amino acid 90-231 (rHaPrP90-231) as the substrate. Under our experimental condition, 37.5\% (6 of 16) of E196A gCJD cases and $60 \%$ (3 of 5) of E196K gCJD cases were positive on CSF RT-QuIC (figure 4 and table 1). There was

B

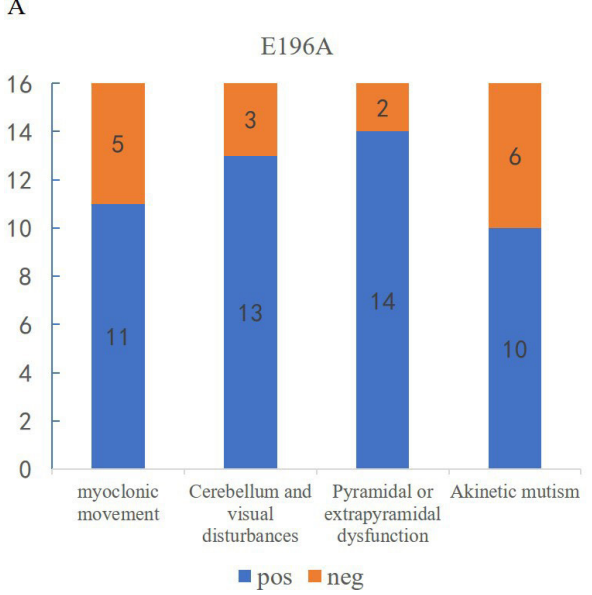

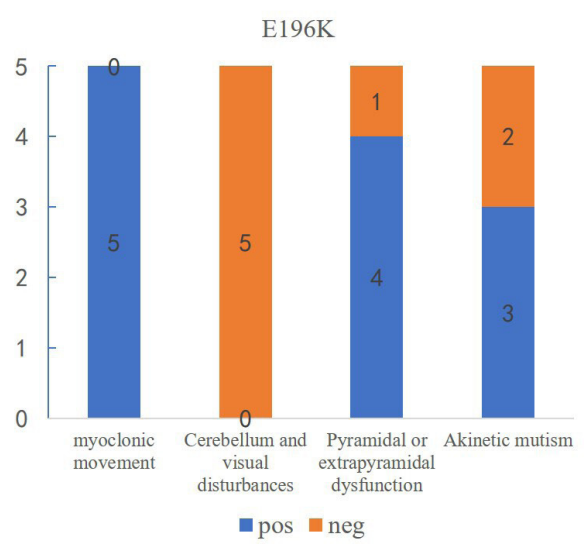

Figure 2 Positive rates and case numbers of four major SCJD-associated symptoms in Chinese patients with (A) E196A and (B) E196K genetic Creutzfeldt-Jakob disease. neg, negative; pos, positive; sCJD, sporadic Creutzfeldt-Jakob disease. 
A

EEG

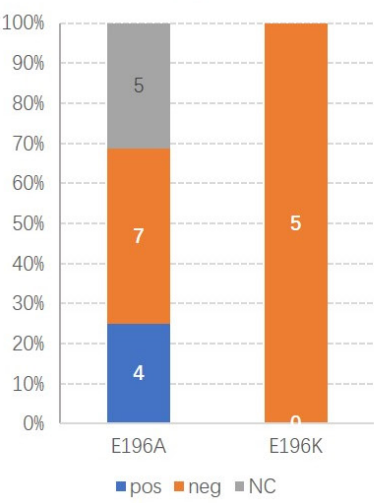

MRI

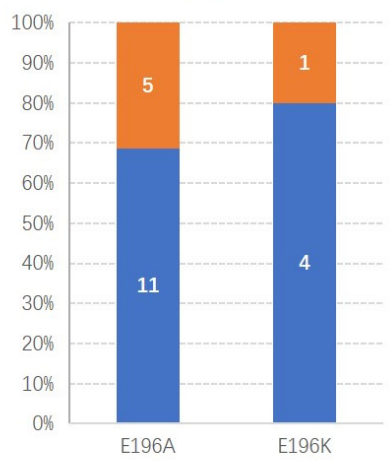

C

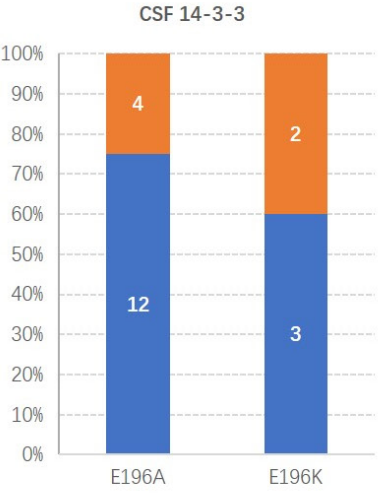

D

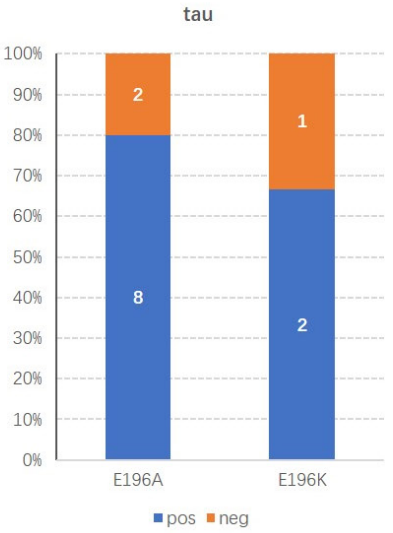

Figure 3 Positive rates and case numbers of various clinical examinations and CSF laboratory tests in Chinese patients with E196A and E196K genetic Creutzfeldt-Jakob disease: (A) PSWC on EEG (B) SCJD-associated abnormality on MRI, (C) CSF 143-3 and (D) CSF total tau. CSF, cerebrospinal fluid; EEG, electroencephalogram; neg, negative; pos, positive; PSWC, periodic sharp wave complex; sCJD, sporadic Creutzfeldt-Jakob disease; NC, uncertain.

no marked difference in the positive conversion time and in the peak of the reactive curves in RT-QuIC between the two groups.

\section{Survival time}

By the end of July 2020, 12 cases with E196A had died, 3 cases were alive and 1 was lost, while 4 cases with E196K had died and 1 was lost (table 1). As shown in figure 5A, the duration of survival of patients with E196A gCJD varied from 2 to 28 months, with a median survival of 6.5 months. Half of the dead cases died within 5 months of onset. All four dead cases of E196K gCJD died within 5 months of onset, with a median of 4.5 months (range $2-5$ months). Analysis of median survival between the two types of gCJD revealed statistical difference $(\mathrm{p}=0.018)$. Three E196A cases were alive, with a clinical duration of 36, 20 and 3 months, respectively (table 1). Further, the survival time of 12 dead cases with E196A was analysed based on gender and age of onset. Male patients $(n=6)$
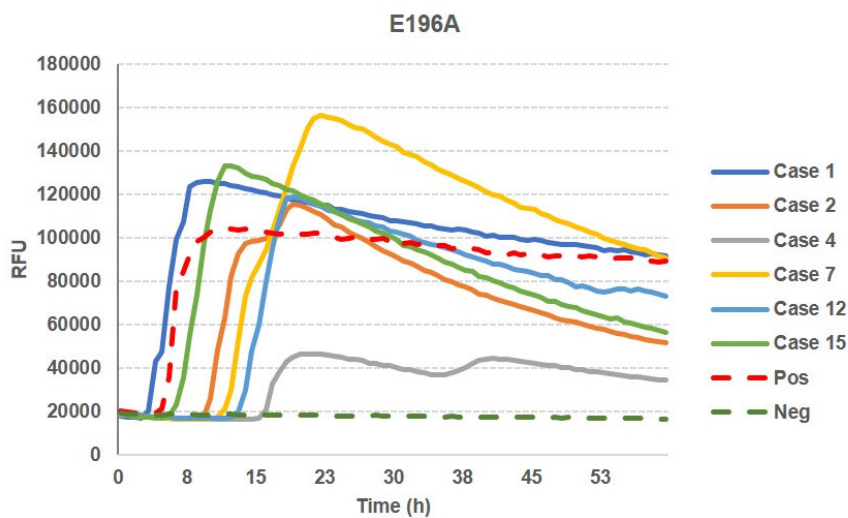

showed much shorter survival time (median: 4 months, range $2-10$ months) than female patients $(n=6)$ (median: 15 months, range 4-28 months) (figure $5 \mathrm{~B}$ ), with significant difference $(p=0.009)$. Senior cases $(\geq 60$ years, $n=7)$ had much shorter survival time (median: 4 months, range $2-8$ months) than the younger ones $(<60$ years, $n=5)$ (figure $5 \mathrm{C}$ ), showing significant difference $(\mathrm{p}=0.001)$.

\section{DISCUSSION}

Human genetic prion diseases display apparent diversity in clinical and laboratory features. In this study, we have comparatively analysed the features of 16 Chinese patients with E196A gCJD and 5 patients with E196K gCJD in terms of demographics, clinical, EEG and MRI, and CSF laboratory tests. Unfortunately, we did not have any brain specimens, either postmortem or biopsy, from the patients so the neuropathological and pathogenic

B

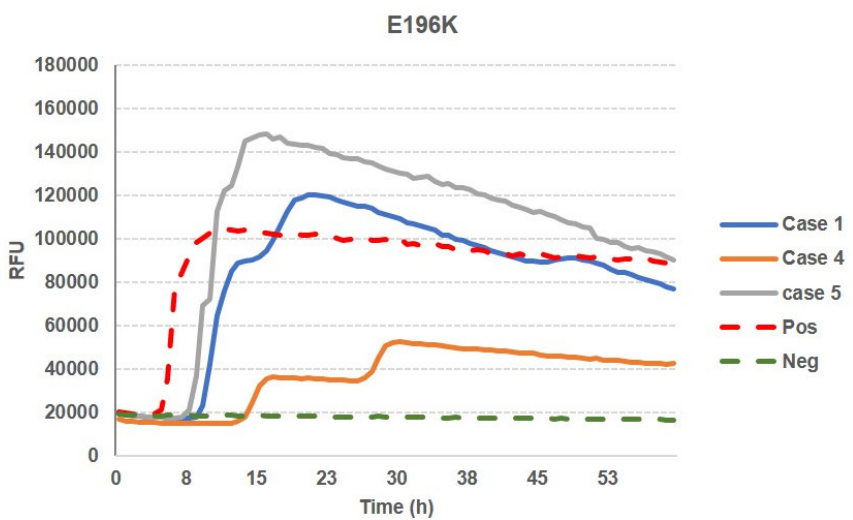

Figure 4 Positive reactive curves of CSF RT-QulC of (A) six E196A patients and (B) three E196K patients with genetic Creutzfeldt-Jakob disease. $10^{-5}$ diluted brain homogenate of scrapie agent $263 \mathrm{~K}$-infected hamster was used as the positive control and that of normal hamster was used as the negative control. ThT value is shown on the $y$-axis and the time post reaction is indicated on the x-axis. CSF, cerebrospinal fluid; neg, negative; pos, positive; RFU, relative fluorescence units. 


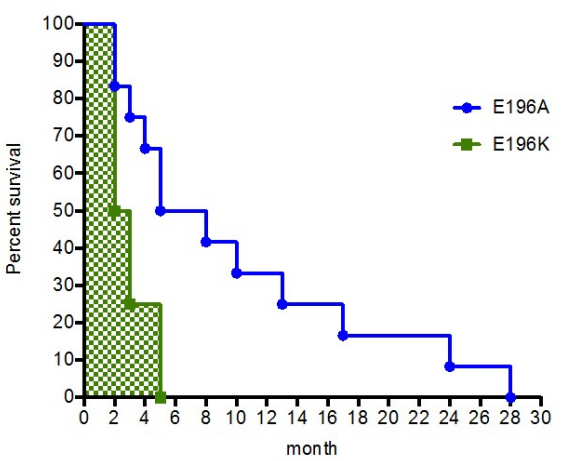

B

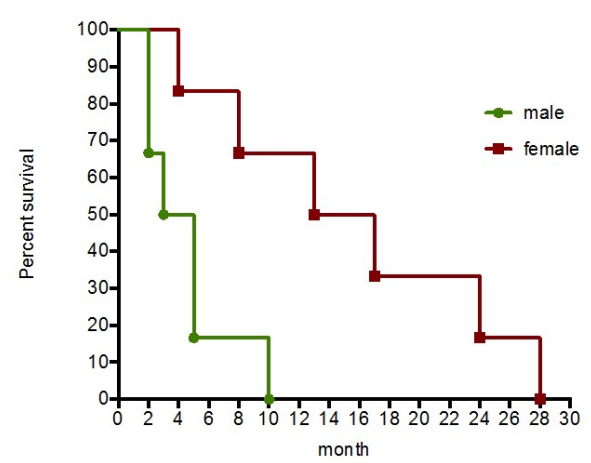

C

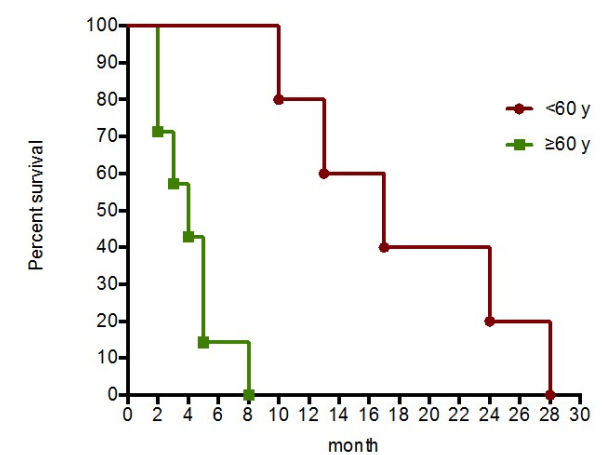

Figure 5 Survival time of Chinese patients with E196A and E196K gCJD. (A) Survival graph of E196A and E196K gCJD cases. (B) Survival graph of E196A cases based on gender. (C) Survival graph of E196A cases based on age of onset. Median survival is indicated in the graphs. gCJD, genetic Creutzfeldt-Jakob disease.

prion protein $\left(\mathrm{PrP}^{\mathrm{Sc}}\right)$ features of Chinese patients with E196A and E196K gCJD remain unclear. Also, there was a limited number of enrolled patients, especially patients with E196K mutation, which may have resulted in observational bias. Generally, the clinical features of both E196A and E196K gCJD cases are like sCJD, for example, displaying rapid progressive dementia and other major manifestations, and high positive rates of MRI abnormalities, CSF 14-3-3 and total tau. However, the E196A and E196K cases in this study also show some degree of differences in some aspects. The age of onset of E196A patients is similar to Chinese patients with $\mathrm{SCJD}^{10}$; however, the five E196K cases were relatively older. E196A cases showed more diverse major symptoms, while symptoms in E196K cases were confined to dementia and mental problems. Cerebellum and visual disturbances were frequent in E196A cases, but not observed in E196K patients. PSWCs on EEG were not observable in all E196K cases but were recordable in a small portion of E196A cases. Additionally, E196K cases seem to have higher ratios of positive CSF RT-QuIC than E196A cases. These observations may be biased due to the small cohort ( $\mathrm{N}=21)$. In fact, E196K gCJD has been reported in many European countries. Unlike the five Chinese cases, the phenotypes in European patients are more diverse, such as having cerebellum problems and PSWC on EEG in a portion of patients. ${ }^{15-18}$ We have to say that majority of the data and specimens of the patients in this study were collected and tested during their last hospitalisation so we cannot exclude the possibility of other neurological signs appearing afterwards. As the number of E196K cases in this study is small, more cases are needed in order to define the difference between Chinese and European E196K gCJD cases.

Four dead cases with E196K displayed much shorter clinical duration than 12 dead E196A cases in this study. However, the survival time of published Caucasian E196K gCJD differs considerably, varying from 2 to 18 months, with a median of 7.5 months. ${ }^{6}{ }^{16-19}$ The cases with E196A in this study showed a wide range of durations, similar to sCJD cases in China. ${ }^{10} 20$ Notably, Chinese female patients and senior patients with E196A gCJD seem to have longer duration of survival than male and young patients in general. More cases are needed to determine the exact feature of survival time in E196 gCJD.

Our data show about three times more E196A cases than E196K cases in the past 10 years. As we do not know the frequency of these two genotypes in general Han Chinese, such diversity in case numbers reflects only the difference in disease occurrence and identification. None of the family members from the 21 patients undertook assays for PRNP sequencing. Thereby, we are also unable to speculate the exact penetrance of these two mutations. The penetrance of mutations in PRNP has been evaluated in several studies. ${ }^{4122}$ However, the exact pathogenicity of many rare mutations remains poorly understood. The penetrance of PRNP mutations is associated with family history. Low-penetrance mutations seem to have low rates of positive family history. ${ }^{21}$ None of the cases in this study recorded family history, which might highlight the low penetrance of E196A and E196K mutations. The penetrance of $P R N P$ mutations is also influenced by age. One example is the Sephardic E200K mutation carrier, the penetrance of which is $70 \%$ at age 70 and close to $100 \%$ at age $85 .{ }^{23}$ Screening PRNP mutations in senior patients with neurological problems will be beneficial in identifying untypical gCJD. On the other hand, relatively late age of onset, such as the Chinese E196K gCJD cases who had a short duration of survival in this study, may also increase the probability of case loss due to death or misdiagnosis of other diseases. Genomic assays, such as whole exome analysis, on patients with E196 mutations in the future may be beneficial in exploring possible modifiable risk factors associated with the disease process.

E196K gCJD was first reported in 2000 and was described in six German patients, ${ }^{16}$ while E196A gCJD was reported late in China. ${ }^{24}$ Based on the literature, dozens of E196K gCJD cases have been described in European countries, such as in Germany, Italy, France and UK. ${ }^{2} 46$ 16-18 25 In contrast, E196K mutation is rarely reported in East Asians, besides the Chinese cases described previously ${ }^{8}$ and in this study. On the other hand, E196A mutation seems to be confined to Chinese and to be extremely rare in other 
countries and ethnicities to date. Again, it shows differences in PRNP mutations and polymorphisms between ethnicities. Nevertheless, E196A gCJD has become the second predominant subtype in Han Chinese after T188K gCJD, ${ }^{910} 26$ which differs not only from Caucasians but also from other East Asians, for example, Japanese and Korean.

\section{CONCLUSION}

E196A gCJD is now the fourth most frequently observed genetic prion disease in China. This is the largest report to date of gCJD with mutations at codon 196. The diversity in clinical and laboratory tests between patients with E196A and E196K mutations indicates that substitutions of different amino acids at the same position with $\operatorname{PrP}$ may associate with different clinical phenotypes.

\section{Author affiliations \\ ${ }^{1}$ State Key Laboratory for Infectious Disease Prevention and Control, Collaborative Innovation Center for Diagnosis and Treatment of Infectious Diseases (Zhejiang University), National Institute for Viral Disease Control and Prevention, Chinese Center for Disease Control and Prevention, Beijing, China \\ ${ }^{2}$ China Academy of Chinese Medical Sciences, Dongzhimeinei, Beijing, China ${ }^{3}$ Center for Biosafety Mega-Science, Chinese Academy of Sciences, Wuhan, China ${ }^{4}$ Center for Global Public Health, Chinese Center for Disease Control and Prevention, Beijing, China}

Acknowledgements We thank colleagues from CDC in all provinces and cities.

Contributors QS contributed to study design, performed the assays and data analysis, and prepared the manuscript. KX, WZ, CG and Y-ZW assisted with the assays of western blot analysis. L-PG, YW, CH and CC assisted with the animal tests. X-PD contributed to design, study concept and manuscript preparation.

Funding This work was supported by Chinese National Natural Science Foundation grants (81630062), as well as grants (2019SKLID501, 2019SKLID603, 2019SKLID307) from the State Key Laboratory for Infectious Disease Prevention and Control, China CDC.

Competing interests None declared.

Patient and public involvement Not Applicable

Patient consent for publication Not required.

Ethics approval Usage of the surveillance data of patients with E196A and E196K gCJD in Chinese National CJD Surveillance System (CNS-CJD) has been approved by the Research Ethics Committee of the National Institute for Viral Disease Control and Prevention, China CDC (CCDC) under protocol 2011ZX10004-101.

Provenance and peer review Not commissioned; externally peer reviewed.

Data availability statement Data are available on reasonable request. All data relevant to the study are included in the article or uploaded as supplementary information.

Open access This is an open access article distributed in accordance with the Creative Commons Attribution Non Commercial (CC BY-NC 4.0) license, which permits others to distribute, remix, adapt, build upon this work non-commercially, and license their derivative works on different terms, provided the original work is properly cited, appropriate credit is given, any changes made indicated, and the use is non-commercial. See: http://creativecommons.org/licenses/by-nc/4.0/.

\section{ORCID iDs}

Qi Shi http://orcid.org/0000-0002-6282-9864

Xiao-Ping Dong http://orcid.org/0000-0002-7022-0418

\section{REFERENCES}

1 Chen C, Dong X-P. Epidemiological characteristics of human prion diseases. Infect Dis Poverty 2016;5:47.
2 Kovács GG, Puopolo M, Ladogana A, et al. Genetic prion disease: the EUROCJD experience. Hum Genet 2005;118:166-74.

3 Baldwin KJ, Correll CM. Prion disease. Semin Neurol 2019;39:428-39.

$4 \mathrm{Kim}$ M-O, Takada LT, Wong K, et al. Genetic PrP prion diseases. Cold Spring Harb Perspect Biol 2018;10:a033134.

5 Jeong B-H, Kim Y-S. Genetic studies in human prion diseases. $J$ Korean Med Sci 2014;29:623-32.

6 Schelzke G, Eigenbrod S, Romero C, et al. Genetic prion disease with codon 196 PRNP mutation: clinical and pathological findings. Neurobiol Aging 2011;32:756.e1-756.e9.

7 Shi Q, Zhou W, Chen C, et al. Rare E196A mutation in PRNP gene of 3 Chinese patients with Creutzfeldt-Jacob disease. Prion 2016;10:331-7.

8 Shi Q, Chen C, Song X-N, et al. A Chinese CreutzfeldtJakob disease patient with E196K mutation in PRNP. Prion 2011;5:117-20.

9 Shi Q, Zhou W, Chen C, et al. The features of genetic prion diseases based on Chinese surveillance program. PLoS One 2015;10:e0139552.

10 Shi Q, Chen C, Zhou W, et al. The characteristics of Chinese prion diseases based on 10 years surveillance data from 2006 to 2015. Neuropsychiatry 2018;8:739-44.

11 Shi Q, Zhang X-C, Zhou W, et al. Analysis of the advantage features of Beijing surveillance network for Creutzfeldt-Jakob disease. Prion 2015;9:304-14.

12 Chen C, Hu C, Shi Q, et al. Profiles of 14-3-3 and total tau in CSF samples of Chinese patients of different genetic prion diseases. Front Neurosci 2019;13:934.

13 Xiao K, Shi Q, Zhou W, et al. T188K-Familial Creutzfeldt-Jacob disease, predominant among Chinese, has a reactive pattern in CSF RT-QulC different from D178N-Fatal familial insomnia and E200KFamilial CJD. Neurosci Bull 2019;35:519-21.

14 Wang G-R, Gao C, Shi Q, et al. Elevated levels of tau protein in cerebrospinal fluid of patients with probable Creutzfeldt-Jakob disease. Am J Med Sci 2010;340:291-5.

15 Eigenbrod S, Frick P, Giese A, et al. Comprehensive neuropathologic analysis of genetic prion disease associated with the E196K mutation in PRNP reveals phenotypic heterogeneity. J Neuropathol Exp Neurol 2011;70:192-200.

16 Peoc'h K, Manivet P, Beaudry P, et al. Identification of three novel mutations (E196K, V203I, E211Q) in the prion protein gene (PRNP) in inherited prion diseases with Creutzfeldt-Jakob disease phenotype. Hum Mutat 2000;15:482.

17 Béjot Y, Osseby G-V, Caillier M, et al. Rare E196K mutation in the PRNP gene of a patient exhibiting behavioral abnormalities. Clin Neurol Neurosurg 2010;112:244-7.

18 Clerici F, Elia A, Girotti F, et al. Atypical presentation of CreutzfeldtJakob disease: the first Italian case associated with E196K mutation in the PRNP gene. J Neurol Sci 2008;275:145-7.

19 Tumani H, Windl O, Kretzschmar HA, et al. [Clinically atypical CJD: diagnostic relevance of cerebrospinal fluid markers and molecular genetic analysis?]. Dtsch Med Wochenschr 2002;127:318-20.

20 Chen C, Wang J-C, Shi Q, et al. Analyses of the survival time and the influencing factors of Chinese patients with prion diseases based on the surveillance data from 2008-2011. PLoS One 2013;8:e62553.

21 Minikel EV, Vallabh SM, Lek M, et al. Quantifying prion disease penetrance using large population control cohorts. Sci Trans/ Med 2016;8:322ra9.

22 Takada LT, Kim M-O, Cleveland RW, et al. Genetic prion disease: experience of a rapidly progressive dementia center in the United States and a review of the literature. Am J Med Genet B Neuropsychiatr Genet 2017;174:36-69.

23 Spudich S, Mastrianni JA, Wrensch M, et al. Complete penetrance of Creutzfeldt-Jakob disease in Libyan Jews carrying the E200K mutation in the prion protein gene. Mol Med 1995;1:607-13.

24 Zhang $\mathrm{H}$, Wang $\mathrm{M}$, Wu L, et al. Novel prion protein gene mutation at codon 196 (E196A) in a septuagenarian with Creutzfeldt-Jakob disease. J Clin Neurosci 2014;21:175-8.

25 Unit, T.N.C.R.S. 27Th annual report 2018, Creutzfeldt-Jakob disease surveillance in the UK. 2018.

26 Shi Q, Zhou W, Chen C, et al. Rare genetic Creutzfeldt-Jakob disease with T188K mutation: analysis of clinical, genetic and laboratory features of 30 Chinese patients. J Neurol Neurosurg Psychiatry 2017;88:889-90. 